\title{
A Note on Galois Cohomology of Algebraic Integers
}

\author{
Takashi ONO \\ Department of Mathematics, The Johns Hopkins University, Baltimore, Maryland, 21218, U.S.A.
}

Received January 7, 2009; final version accepted March 6, 2009

\begin{abstract}
We consider an admissible system $(\mathfrak{g},(G, M))$ where $\mathfrak{g}$ is the group of a Galois extension $k / \mathbf{Q}$ of number fields, $G$ the ring $\mathcal{O}_{k}$ of integers of $k$ and $M=\left(\mathcal{O}_{k}\right)^{2}$. The cohomology group $H^{1}\left(\mathfrak{g}, \mathcal{O}_{k}\right)$ is nicely described by a naive submodule $\Xi_{k}$ of $\mathcal{O}_{k}$ and the Poincaré index $i_{\gamma}(\mathfrak{g}, M), \gamma \in H^{1}\left(\mathfrak{g}, \mathcal{O}_{k}\right)$, for the system $(\mathfrak{g},(G, M))$, can be expressed in terms of $\Xi_{k}$.
\end{abstract}

KEYWORDS: Galois extension, number fields, ring of integers, cohomology, system

\section{The module $\Xi_{k}$}

Let $k / \mathbf{Q}$ be a finite Galois extension. Denote by $\mathfrak{g}$ the Galois group $\operatorname{Gal}(k / \mathbf{Q})$ and by $d$ the degree $|\mathfrak{g}|=[k: \mathbf{Q}]$. Let $\mathcal{O}_{k}$ be the ring of integers in $k$. We set

$$
\Xi_{k}=\left\{\xi \in \mathcal{O}_{k} ;{ }^{\sigma} \xi \equiv \xi \bmod d, \forall \sigma \in \mathfrak{g} .\right\}
$$

This is a $\mathbf{Z}$-module in $\mathcal{O}_{k}$. It contains $\mathbf{Z}$ and $d \mathcal{O}_{k}$, and is $\mathfrak{g}$-stable as is easily seen. The definition implies that

$$
\Xi_{k} / d \mathcal{O}_{k}=\left(\mathcal{O}_{k} / d \mathcal{O}_{k}\right)^{\mathfrak{g}},
$$

where, for any $\mathfrak{g}$-module $M, M^{\mathfrak{g}}$ denotes the subset of elements of $M$ invariant under the action of $\mathfrak{g}$.

For each $\xi \in \Xi_{k}$ and $\sigma \in \mathfrak{g}$, we can define an element $t(\xi)_{\sigma}$ in $\mathcal{O}_{k}$ by

$$
t_{\sigma}=t(\xi)_{\sigma}=\left(\xi-{ }^{\sigma} \xi\right) / d .
$$

As the group $\mathfrak{g}$ acts on the additive group of the ring $\mathcal{O}_{k}$, we may speak of the first cohomology group $H^{1}\left(\mathfrak{g}, \mathcal{O}_{k}\right)=Z^{1}\left(\mathfrak{g}, \mathcal{O}_{k}\right) / B^{1}\left(\mathfrak{g}, \mathcal{O}_{k}\right)$. In view of (3), we have

$$
t_{\sigma \tau}=t_{\sigma}+{ }^{\sigma} t_{\tau}
$$

and hence a homomorphism

$$
t: \Xi_{k} \rightarrow Z^{1}\left(\mathfrak{g}, \mathcal{O}_{k}\right), \text { defined by } t(\xi)_{\sigma}=\left(\xi-{ }^{\sigma} \xi\right) / d \text {. }
$$

Proposition 1. The map $t$ in (5) induces an isomorphism

$$
\Xi_{k} / \mathbf{Z} \approx Z^{1}\left(\mathfrak{g}, \mathcal{O}_{k}\right) .
$$

Proof. (i) $\operatorname{Ker} t=\mathbf{Z}$. In fact,

$$
\xi \in \operatorname{Ker} t \Longleftrightarrow t(\xi)=0 \Longleftrightarrow \xi-{ }^{\sigma} \xi=0, \sigma \in \mathfrak{g} \Longleftrightarrow \xi \in \mathcal{O}_{k} \cap \mathbf{Q}=\mathbf{Z} .
$$

(ii) $t$ is surjective. Let $u$ be any cocycle in $\mathbf{Z}^{1}\left(\mathfrak{g}, \mathcal{O}_{k}\right)$. We will find a $\xi \in \Xi_{k}$ so that $u=t(\xi)$. Let us try the following element

$$
\xi=\sum_{\tau \in \mathfrak{g}} u_{\tau} \in \mathcal{O}_{k} .
$$

It is not obvious at this moment that $\xi \in \Xi_{k}$ but the following argument implies it. In fact, we have

$$
\left(\xi-{ }^{\sigma} \xi\right) / d=\left(\sum_{\tau} u_{\tau}-\sum_{\tau}{ }^{\sigma} u_{\tau}\right) / d=\left(\sum_{\tau} u_{\sigma \tau}-\sum_{\tau}{ }^{\sigma} u_{\tau}\right) / d=\left(\sum_{\tau} u_{\sigma}\right) / d=u_{\sigma}
$$

which shows that our $\xi \in \Xi_{k}$ and $u=t(\xi)$.

From Proposition 1 we have the relation: 


$$
\mathcal{O}_{k} / \mathbf{Z} \supset \Xi_{k} / \mathbf{Z} \approx Z^{1}\left(\mathfrak{g}, \mathcal{O}_{k}\right) .
$$

Let $H$ be the subgroup of $\Xi_{k} / \mathbf{Z}$ corresponding to the coboundary group:

$$
B^{1}\left(\mathfrak{g}, \mathcal{O}_{k}\right)=\left\{v \in Z^{1}\left(\mathfrak{g}, \mathcal{O}_{k}\right) ; v_{\sigma}=\eta-{ }^{\sigma} \eta, \eta \in \mathcal{O}_{k}\right\} .
$$

Denoting by $[\xi]$ the class of $\xi \in \mathcal{O}_{k}$ modulo $\mathbf{Z}$, we have, for $\xi \in \mathbf{\Xi}_{k}$,

$$
[\xi] \in H \Longleftrightarrow t(\xi)_{\sigma}=\left(\xi-{ }^{\sigma} \xi\right) / d=\eta-{ }^{\sigma} \eta, \eta \in \mathcal{O}_{k} \Longleftrightarrow \xi-d \eta={ }^{\sigma}(\xi-d \eta) \Longleftrightarrow \xi-d \eta \in \mathbf{Z} .
$$

In other words,

$$
H=d\left(\mathcal{O}_{k} / \mathbf{Z}\right)=\left(\mathbf{Z}+d \mathcal{O}_{k}\right) / \mathbf{Z}
$$

By (6), (7), (8), (9), we obtain

Proposition 2. Let $k / \mathbf{Q}$ be a finite Galois extension of degree $d$ with the Galois group $\mathfrak{g}$ and let $\Xi_{k}$ be the module given by (1). Then we have

$$
H^{1}\left(\mathfrak{g}, \mathcal{O}_{k}\right) \approx \Xi_{k} /\left(\mathbf{Z}+d \mathcal{O}_{k}\right)
$$

We note here an obvious relation:

$$
\mathcal{O}_{k} / d \mathcal{O}_{k} \approx(\mathbf{Z} / d \mathbf{Z})^{d} .
$$

In connection with the cohomology group $H^{1}\left(\mathfrak{g}, \mathcal{O}_{k}\right)$, we will study the $\mathfrak{g}$-fixed points of the finite module $\mathcal{O}_{k} / d \mathcal{O}_{k}$. Let us look at the sequence of modules:

$$
\mathcal{O}_{k} \supset \Xi_{k} \supset \mathbf{Z}+d \mathcal{O}_{k} \supset d \mathcal{O}_{k}
$$

We already know structures of several portions of (11). By (10), we have

$$
\left(\mathcal{O}_{k}: d \mathcal{O}_{k}\right)=d^{d},
$$

by (2)

$$
\left(\Xi_{k}: d \mathcal{O}_{k}\right)=\left|\left(\mathcal{O}_{k} / d \mathcal{O}_{k}\right)^{\mathfrak{g}}\right|
$$

by Proposition 2

$$
\left(\Xi_{k}: \mathbf{Z}+d \mathcal{O}_{k}\right)=\left|H^{1}\left(\mathfrak{g}, \mathcal{O}_{k}\right)\right| .
$$

Combining (12), (13), (14) with the obvious isomorphism

$$
\left(\mathbf{Z}+d \mathcal{O}_{k}\right) / d \mathcal{O}_{k} \approx \mathbf{Z} / d \mathbf{Z},
$$

we obtain

Theorem 1. Let $k / \mathbf{Q}$ be a finite Galois extension of degree $d$ with the Galois group $\mathfrak{g}$. Then we have

$$
\left|H^{1}\left(\mathfrak{g}, \mathcal{O}_{k}\right)\right|=\left|\left(\mathcal{O}_{k} / d \mathcal{O}_{k}\right)^{\mathfrak{g}}\right| / d
$$

\section{The module $\left(\mathcal{O}_{k} / d \mathcal{O}_{k}\right)^{\mathfrak{g}}$}

Notations being as before, let us express an element $\xi \in \mathcal{O}_{k}$ as

$$
\xi=x_{1} \omega_{1}+\cdots+x_{d} \omega_{d}=\Omega x, \quad \Omega=\left(\omega_{1}, \cdots, \omega_{d}\right),
$$

where $\left\{\omega_{i}\right\}$ being a $\mathbf{Z}$-basis of $\mathcal{O}_{k}$ with

$$
x=\left(\begin{array}{c}
x_{1} \\
\vdots \\
x_{d}
\end{array}\right) \in \mathbf{Z}^{d} .
$$

Then

$$
{ }^{\sigma} \xi={ }^{\sigma} \Omega x, \quad \sigma \in \mathfrak{g} .
$$

If we define a unimodular matrix $A_{\sigma}$ by

$$
{ }^{\sigma} \Omega=\Omega A_{\sigma}
$$

then, we have 


$$
A_{\sigma \tau}=A_{\sigma} A_{\tau}, \quad \sigma, \tau \in \mathfrak{g} .
$$

In other words,

$$
A: \quad \sigma \mapsto A_{\sigma} \in G L_{d}(\mathbf{Z})
$$

is an integral representation of the Galois group $\mathfrak{g}$ of degree $d=[k: \mathbf{Q}]$.

As for the base change $\Omega \rightarrow \Omega^{\prime}$ afforded by $\Omega^{\prime}=\Omega T, T \in G L_{d}(\mathbf{Z})$, let $A_{\sigma}^{\prime}$ be the matrix for the new basis. One then finds

$$
A_{\sigma}^{\prime}=T^{-1} A_{\sigma} T
$$

so $A^{\prime}$ is $\mathbf{Z}$-equivalent to $A: A^{\prime} \sim A$.

Let $\xi=\Omega x$ be the relation in (15). We want to translate the condition $\xi \in \Xi_{k}$ in terms of one for $x \in \mathbf{Z}^{d}$. Now follow the following sequence of equivalences

$$
\xi \in \Xi_{k} \Leftrightarrow{ }^{\sigma} \xi \equiv \xi(d) \Leftrightarrow{ }^{\sigma} \Omega x \equiv \Omega x(d) \Leftrightarrow \Omega A_{\sigma} x \equiv \Omega x(d) \Leftrightarrow\left(A_{\sigma}-I\right) x \equiv 0(d) .
$$

Therefore we obtain the following theorem:

Theorem 2. Notations being as above, we have

$$
\left|\left(\mathcal{O}_{k} / d \mathcal{O}_{k}\right)^{\mathfrak{g}}\right|=\left|\left\{x \in \mathbf{Z}^{d} / d \mathbf{Z}^{d} ;\left(A_{\sigma}-I\right) x \equiv 0 \bmod d\right\}\right| .
$$

\section{Case $d=2$}

Let us study here a special case where $d=|\mathfrak{g}|=[k: \mathbf{Q}]=2$. Let $\sigma$ be the generator of $\mathfrak{g}$. As we know there is an $\Omega$ of the form $\Omega=(1, \omega)$. [In general, it is true that 1 can appear in a basis of $\mathcal{O}_{k}$ for any number field $k$.] Then the matrix $A_{\sigma}, \sigma \neq 1$, satisfying (16) must be of the form

$$
\left(\begin{array}{cc}
1 & b \\
0 & -1
\end{array}\right)
$$

Furthermore it is easy to check that the parity of $b$ in (18) is unchanged by the base change $\Omega=(1, \omega) \rightarrow \Omega^{\prime}=\left(1, \omega^{\prime}\right)$. We shall call the parity of $b$ simply the parity of the quadratic field $k$. Let us set $p(k)=1$ or 2 according as the parity is odd or even. Theorem 1 and Theorem 2, with elementary facts on quadratic fields, imply the following

Proposition 3. Let $k / \mathbf{Q}$ be a quadratic field. Then

$$
p(k)=\left|H^{1}\left(\mathfrak{g}, \mathcal{O}_{k}\right)\right|=\frac{1}{2}\left|H^{0}\left(\mathfrak{g}, \mathcal{O}_{k} / 2 \mathcal{O}_{k}\right)\right| .
$$

\section{The system $(\mathfrak{g},(G, M))$}

To begin with let us introduce a simple general setting

$$
(\mathfrak{g},(G, M))
$$

where $G$ is a group, $M$ a left $G$-module and $\mathfrak{g}$ is a finite group acting on $(G, M)$ naturally. Namely, we assume that $G$ is a left $\mathfrak{g}$-group, $M$ is a left $\mathfrak{g}$-module so that

$$
{ }^{\sigma}(s x)={ }^{\sigma} s^{\sigma} x, \quad \sigma \in \mathfrak{g}, s \in G, x \in M .
$$

For a cocycle $C$ of $\mathfrak{g}$ with values in $G$, we associate a subgroup $M_{C}$ of $M$ by

$$
M_{C}=\left\{x \in M ; C_{\sigma}{ }^{\sigma} x=x, \quad \sigma \in \mathfrak{g}\right\} .
$$

We consider also a subgroup of $M_{C}$ given by

$$
P_{C}=\left\{y=p_{C}(x), \quad x \in M\right\}
$$

where

$$
p_{C}(x)=\sum_{\tau \in \mathfrak{g}} C_{\tau}^{\tau} x .
$$

One verifies that the structure of the module $M_{C} / P_{C}$ depends only on the cohomology class $\gamma=[C] \in H^{1}(\mathfrak{g}, G)$. If we put $C=1$ in (19), (20) then we have $M_{1}=M^{\mathfrak{g}}, P_{1}=N(M)$ hence $M_{1} / P_{1}=\hat{H}^{0}(\mathfrak{g}, M)$, the Tate cohomology group. For a general $\gamma=[C] \in H^{1}(\mathfrak{g}, G)$, we have a right to make identification 


$$
M_{C} / P_{C}=\hat{H}^{0}(\mathfrak{g}, M)_{\gamma}
$$

and call this the Tate group twisted by $\gamma$. Furthermore, we shall put

$$
i_{\gamma}(\mathfrak{g}, M)=\left[M_{C}: P_{C}\right], \quad \gamma=[C] \in H^{1}(\mathfrak{g}, G) .
$$

The determination of the index $i_{\gamma}(\mathfrak{g}, M)$ is a theme inspired by Poincaré. (See [1], Appendix 3 (in English) and references there.)

Now Let $k / \mathbf{Q}$ be a finite Galios extension with the Galois group $\mathfrak{g}$. As for the group $G$ and the module $M$, we set $G=\left\{g=\left(\begin{array}{c}1 t \\ 01\end{array}\right), t \in \mathcal{O}_{k}\right\}, \quad M=\left\{x=\left(\begin{array}{c}x_{1} \\ r_{1}\end{array}\right), \quad x_{1}, x_{2} \in \mathcal{O}_{k}\right\}$.

With the natural action of $G$ on $M$ and those of $\mathfrak{g}$ on each of $g$ and $M$, we obtain an admissible system $(\mathfrak{g},(G, M))$. The

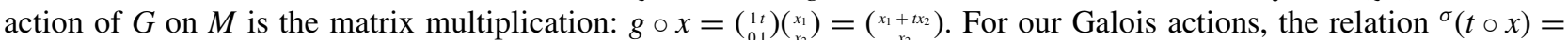
$\left({ }^{\sigma} t\right) \circ\left({ }^{\sigma} x\right)$ is trivial. We can use matrices $C_{\sigma}=\left(\begin{array}{c}1_{01} t \\ 01\end{array}\right), \sigma \in \mathfrak{g}$ as a cocycle $\in Z^{1}(\mathfrak{g}, G)$. Instead of additive relation (4), we have, this time, a multiplicative one: $C_{\sigma \tau}=C_{\sigma}{ }^{\sigma} C_{\tau}$. Since any cocycle $t \in Z^{1}\left(\mathfrak{g}, \mathcal{O}_{k}\right)$ is of the form $t_{\sigma}=t(\xi)_{\sigma}=$ $\left(\xi-{ }^{\sigma} \xi\right) / d$ for some $\xi \in \Xi_{k}$ by (6), we can write

$$
C_{\sigma}=A(\xi) \cdot{ }^{\sigma} A(\xi)^{-1}
$$

with the matrix

$$
A(\xi)=\left[\begin{array}{cc}
1 & \xi / d \\
0 & 1
\end{array}\right] .
$$

Along with (19), (20), for the cocycle $C$, we associate a $\mathbf{Z}$-module $M_{C}$ by

$$
M_{C}=\left\{x \in M ; C_{\sigma}{ }^{\sigma} x=x, \sigma \in \mathfrak{g}\right\} .
$$

We have also a submodule of $M_{C}$ given by

$$
P_{C}=\left\{y=p_{C}(x), x \in M\right\}
$$

where $p_{C}(x)=\sum_{\tau} C_{\tau}^{\tau} x$.

We know that the quotient $M_{C} / P_{C}$ depends only on the cohomology class $\gamma=[t]=[t(\xi)] \in H^{1}\left(\mathfrak{g}, \mathcal{O}_{k}\right)$ and is identified with the module $\hat{H}^{0}(\mathfrak{g}, M)_{\gamma}$. Finally we set index $i_{\gamma}(\mathfrak{g}, M)=\left[M_{C}: P_{C}\right]$.

In what follows, when a $\xi \in \Xi_{k}$ is fixed, we set simply $A=A(\xi)$.

(i) $M_{C}$. For $x \in M$,

$$
x \in M_{C} \Longleftrightarrow C_{\sigma}{ }^{\sigma} x=x \Longleftrightarrow A^{\sigma} A^{-1 \sigma} x=x \Longleftrightarrow A^{-1} x={ }^{\sigma}\left(A^{-1} x\right) .
$$

Hence we have

$$
A^{-1} M_{C}=\left\{A^{-1} x \in A^{-1} M \cap(\mathbf{Q})^{2}\right\} .
$$

In other words,

$$
A^{-1} M_{C}=\left(A^{-1} M\right)^{\mathfrak{g}} .
$$

(ii) $P_{C}$. By (20), we find

$$
A^{-1} P_{C}=\left\{A^{-1} \sum_{\tau} C_{\tau}{ }^{\tau} x\right\}=\left\{\sum_{\tau}{ }^{\tau}\left(A^{-1} x\right)\right\}=\left\{\operatorname{Tr}\left(A^{-1} x\right), x \in M\right\} .
$$

In other words,

$$
A^{-1} P_{C}=\operatorname{Tr}\left(A^{-1} M\right)
$$

By (24), (25) we obtain

Theorem 3. Let $k / \mathbf{Q}$ be a finite Galois extension with the Galois group $\mathfrak{g}$. Let $M=\left(\mathcal{O}_{k}\right)^{2}$. For a cocycle class $\gamma=[C] \in H^{1}\left(\mathfrak{g}, \mathcal{O}_{k}\right)$ corresponding to an element $\xi \in \Xi_{k}$ by $(6)$, we have $i_{\gamma}(\mathfrak{g}, M)=\left|\hat{H}^{0}\left(\mathfrak{g}, A(\xi)^{-1} M\right)\right|$.

\section{REFERENCES}

[1] Ono, T., Gauss sums and Poincaré sums (in Japanese), Nippon Hyoronsha, Tokyo (2008). 